\title{
Phase of harmonics from strongly driven two-level atoms
}

\author{
F. I. Gauthey, ${ }^{1,2}$ C. H. Keitel, ${ }^{1}$ P. L. Knight, ${ }^{1}$ and A. Maquet ${ }^{2}$ \\ ${ }^{1}$ Optics Section, Blackett Laboratory, Imperial College, London SW7 2BZ, England \\ ${ }^{2}$ Laboratoire de Chimie-Physique-Matière et Rayonnement, Université Pierre et Marie Curie, Paris, France
}

(Received 10 June 1996)

\begin{abstract}
Harmonic generation is studied in an idealized model medium in which the laser field essentially interacts only with a single atomic transition and where ionization can be neglected. Such two-level models are known to generate high harmonics with a plateau and a cutoff. We put forward a quantitative rule for the cutoff frequency in the harmonic spectrum in this model which we relate to the " $I_{P}+3 U_{P}$ rule" in real systems with ionization. We examine the phase properties of the emitted harmonics and show that their coherence and thus phase matching is more favorable in the cutoff regime than in the plateau of the harmonic spectrum. This behavior is known to occur in realistic models of neon, and that this occurs also for the simple idealized two-level model strongly suggests that the sudden change of phase coherence from the cutoff to the plateau regime is as intrinsic a property of nonlinear systems as are the plateau and the cutoff. [S1050-2947(97)03401-X]
\end{abstract}

PACS number(s): 42.50.Hz, 42.65.Ky, 32.80.Wr

\section{INTRODUCTION}

In the field of harmonic generation from the interaction of atoms with intense laser fields, single atom theoretical models [1-6] have been very successful in predicting the main features of the spectrum. These are (a) a rapid decrease in the perturbative part of the harmonic spectrum, (b) a successive plateau regime of harmonics with approximately constant intensity, and finally (c) the cutoff region with an abruptly decreasing harmonic intensity. This behavior has proven generic for all theoretical models of high harmonic generation presently studied, including in particular the strongly driven two-level system [7-13]. In the case of model atoms which allow ionization, a widely accepted interpretation of the cutoff energy (in the tunneling regime) relates the highest radiated frequency to the maximum kinetic energy of approximately three times the ponderomotive energy $U_{P}$ plus the ionization energy $I_{P}$, which the ionized electron optimally releases when it returns from the continuum and recombines with the atomic core to reemerge in the ground state [14]. In the case of a two-level system where no ionization is possible, Sundaram and Milonni [7], Kaplan and Shkolnikov [12], and Ivanov and Corkum [15] have proposed a linear dependence of the cutoff harmonic order on the Rabi frequency of the driving laser field in the intense field limit. For the entire multiatom ensemble, which is of course of more relevance for experimental realizations [16,17], phase matching among the harmonics from the various atoms becomes an important issue. For this reason experimental observation of high harmonics is implemented in the forward direction of the laser beam, where in principle phase matching is essentially perfect given that the laser is assumed to have nearperfect coherence and has a constant intensity over the spread of the atomic sample [10]. However, in reality, the necessity to focus the laser beam implies a variable intensity dependence. Under these circumstances, the intensity dependence of the phases of the harmonics becomes relevant again even for forward detection. Very recently Lewenstein and co-workers have addressed this problem $[18,19]$, and showed that the intensity dependence of the phase of the harmonics of laser driven neon atoms is substantially stronger in the plateau regime than in the cutoff regime.

In this paper we investigate the phase coherence of harmonic generation of strongly driven two-level systems and present results which are qualitatively very similar to those of Refs. $[18,19]$ for neon although quantitative differences exist due to the differing parameters of our system. This strongly suggests that the improved phase coherence of harmonics in the cutoff regime is a generic property of the interaction of strong laser fields with nonlinear systems.

In the following preliminary considerations (Sec. II) we define the central concepts and the main working expressions, and summarize the results of Salières et al. [18]. This is followed by a presentation of the two-level model in Sec. III. Then in Sec. IV we display typical harmonic spectra and put forward and interpret a rule for the two-level cutoff frequency as a function of the atomic transition frequency and the Rabi frequency of the driving field. Before concluding we determine in Sec. V the phase of various harmonics as a function of the Rabi frequency and relate this behavior to that of the corresponding stationary states in the Floquet picture. Finally in Sec. V, we compare the slopes of this phase dependence in the plateau and the cutoff regime and make comments on its implications for phase matching.

\section{PRELIMINARY CONSIDERATIONS}

In the recent study of Salières, L'Huillier, and Lewenstein [18] on the conditions for optimal coherence of harmonics from strongly driven atoms, it was shown that the variations of the phase of the atomic dipole moment due to the focusing geometry affect dramatically the spatial and spectral coherence of the generated harmonics. Their theoretical approach, based on an atomic model consisting of a single bound state coupled to a flat continuum, is that developed in [5]; see also for an alternative viewpoint [6]. The single atom spectrum 
considered here is the "coherent" part of the total spectrum, that is, the spectrum of the mean dipole (see [13], and references therein):

$$
S_{C}(\omega)=|d(\omega)|^{2}
$$

where $d(\omega)$ is defined as

$$
d(\omega)=\int d t e^{i \omega t}\langle x(t)\rangle
$$

We use here the dipole form rather than the more correct dipole acceleration [20] to describe the spectrum, as in the absence of ionization these differ only by unimportant multiplicative constants. The dipole moment $d_{q}$ at the $q$ th harmonic frequency $q \omega_{L}$ is hence given by

$$
d_{q}=\int d t e^{i q \omega_{L} t}\langle x(t)\rangle=r_{q} e^{i \varphi_{q}}
$$

and we define $\varphi_{q}$ as the phase of a particular harmonic of order $q$, and $r_{q}$ as its amplitude. The $q$ th component of the dipole, $d_{q}$, is a function which depends in particular upon whether the harmonic belongs to the "cutoff"' region [located at $q_{\max } \simeq\left(I_{p}+3 U_{p}\right) / \omega_{L}$ with $U_{p}=E_{0}^{2} / 4 \omega_{L}^{2}$ for the realistic atomic models in the tunneling regime, see $[5,14]]$, or to the "plateau.',

The study performed in [18] consisted of looking at the intensity dependence of the phase of the dipole moment $\varphi_{q}$, at a particular harmonic, in the cutoff and the plateau region. It revealed that in the cutoff region, the phase decreases linearly as $\sim-3.2 U_{p} / \omega_{L}$, whereas in the plateau region it varies essentially linearly but more rapidly (as $\left.\sim-5.8 U_{p} / \omega_{L}\right)$. These dependences are believed to be related to the time needed for the electron to return to the nucleus during the recombination process [19]. An immediate conclusion concerning the phase dependence of the $q$ th-harmonic dipole component upon the field intensity is that the best conditions for phase matching for harmonics are obtained when the intensity in the medium is such that the harmonics are in the cutoff regime.

We are here interested in relating these results to those obtained from the case of a model in which ionization does not exist, and hence for which the recombination theory (and thus the argument of different return times leading to different slopes) is not applicable. We present here results obtained for a strongly driven two-level system. Surprisingly, perhaps, we come to strikingly similar conclusions provided $I_{P}$ and $U_{P}$ are replaced by transition frequencies and Rabi frequencies. We use a semiclassical model and perform numerical calculations beyond the rotating-wave approximation [referred to as RWA from now on (for details see [13])]. We first define an appropriate cutoff law formula for the twolevel system in the strong field limit; this empirical formula is expressed in terms of the relevant parameters for the case of a two-level system and allows us to define the cutoff and plateau region for each harmonic. We then study the evolution of the phase of a particular high harmonic as a function of the Rabi frequency. The paper ends with conclusions.

\section{TWO-LEVEL MODEL}

We consider a two-level system where $|g\rangle$ and $|e\rangle$ represent the ground and excited states, respectively, with corresponding energies $E_{g}$ and $E_{e}$. We denote $\omega_{0}=\left(E_{e}-E_{g}\right) / \hbar$ as the atomic frequency between the two levels. We assume the laser driving field to be in a coherent state so that we can adopt a semiclassical model in which the quantum atomic system interacts with the classical electric field $\mathbf{E}=\mathbf{E}_{0} \sin \left(\omega_{L} t\right)$ with $\omega_{L}$ being the laser frequency and $\mathbf{E}_{0}$ its amplitude, orientated along the polarization axis $\hat{\boldsymbol{\epsilon}}_{x}$. This model is furthermore justified as we only consider the spectrum radiated by the source, which remains unchanged if we replace the quantized driving field by an approximate classical field. The interaction Hamiltonian in the dipole approximation is

$$
H_{I}=-\mathbf{d} \cdot \mathbf{E}(t)=\hbar \Omega_{0} \sin \left(\omega_{L} t\right) .
$$

d is the quantum dipole operator which is expressed in terms of the atomic transition operators $\sigma_{n m}=|n\rangle\langle m|$ : $\mathbf{d}=x \boldsymbol{\epsilon}_{x}=\mu\left(\sigma_{g e}+\sigma_{e g}\right) \boldsymbol{\epsilon}_{x}$ with $\mu=\mu_{g e}=\mu_{e g}$ the electricdipole transition matrix element between the two states and $\boldsymbol{\epsilon}_{x}$ the unit polarization vector of the dipole, taken to be orientated along the direction of polarization of the laser field. We have introduced in the above expression (4) the Rabi frequency $\Omega_{0}=-\mathbf{E}_{0} \cdot \mu \boldsymbol{\epsilon}_{x} / \hbar$, and will also denote $\Delta=\omega_{0}-\omega_{L}$ as the detuning between the atomic and the laser frequencies. All calculations are performed numerically beyond the RWA (see [13]).

The coherent spectrum, or spectrum of the mean dipole, is expressed as

$$
S_{C}(\omega)=\left|\int d t e^{i \omega t}\langle x(t)\rangle\right|^{2}=|d(\omega)|^{2},
$$

where $x(t)$ represents the dipole operator in the Heisenberg picture: $x(t)=U^{\dagger}(t) x U(t)$ with $U(t)$ being the evolution operator of the system, and $x$ the dipole operator prior to the application of the laser pulse. The intensity of harmonics $\left|d_{q}\right|^{2}$ as a function of the order $q$ is presented in Fig. 1, for some particular driving frequencies and Rabi frequencies, when the system is initially prepared in its ground state. All calculations were performed with square laser pulses of 64 cycles. The spectra show similar features as those observed in the case of real atoms, i.e., a rapid decrease in harmonic intensity, followed by a plateau and a cutoff. The full spectra also show sidebands [hyper-Raman lines (see [13])] which are here manually suppressed in these figures for clarity. The positions and lengths of the regimes depend sensitively on the parameters we use, i.e., the Rabi frequency of the driving field, the energy difference between the two levels, and the detuning of the laser frequency from atomic resonance. Comparing Figs. 1(a) and 1(b) we notice that the plateau increases with increasing Rabi frequency. We also find that an increased laser frequency with respect to that in Fig. 1(a), and therefore a reduced detuning, leads to an increased efficiency in harmonic generation, in the sense that the harmonic peaks have a higher intensity. Nevertheless, the length of the plateau, as evaluated here as a function of the harmonic or- 

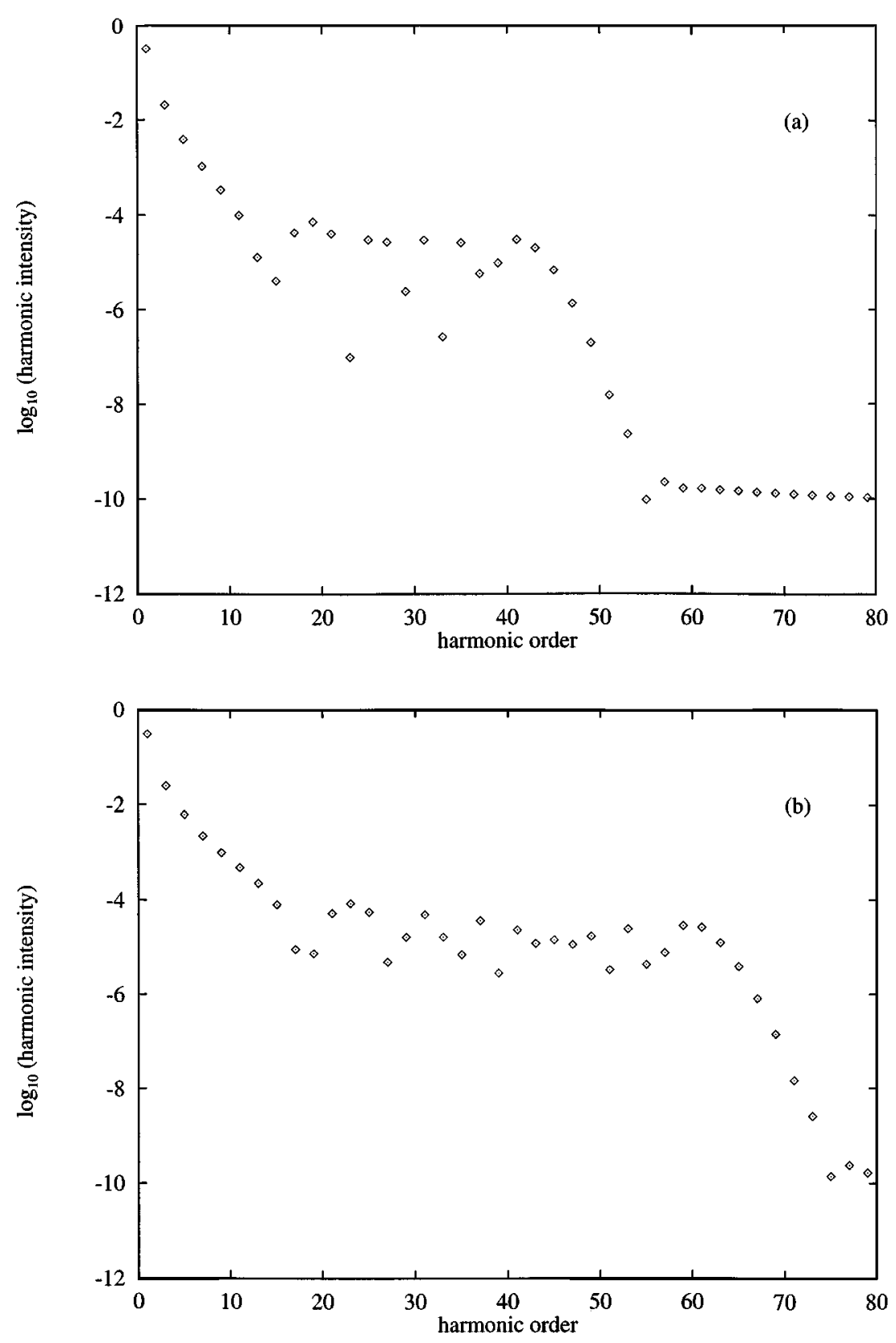

FIG. 1. The logarithm of the harmonic intensities $\left|d_{q}\right|^{2}$ as a function of their order $q$ is shown under different conditions of excitation from the ground level of the system. The Fourier transforms are computed for pulses that are 64 cycles of the laser frequency in duration, and the frequency unit is the laser frequency. The atomic transition frequency is equal to $\omega_{0}=0.3$ a.u. and the laser frequency to $\omega_{L}=0.0258$ a.u. The Rabi frequencies are, respectively, equal to $\Omega_{0}=0.55$ a.u. (a) and $\Omega_{0}=0.8$ a.u. (b). All units are in atomic units if not specified otherwise. der, is decreased by a factor of about 2 when the laser frequency is doubled. This means that the cutoff frequency, i.e., the "cutoff" harmonic order times the laser frequency, is essentially unchanged. We can relate this to the fact that $U_{P}$, which is a function of the square of the laser frequency, cannot play a role in the expression of the cutoff frequency in the case of a two-level atom (this will be discussed in Sec. IV). We also found finally that the increase of the atomic frequency yields a slightly extended plateau for the relatively large Rabi frequencies that we have applied here, however, with smaller harmonic intensities. This indicates that the atomic frequency $\omega_{0}$ contributes to the expression for the cutoff frequency.

\section{CUTOFF LAW FORMULA}

We have noted from Fig. 1, as is well known, that there is a final harmonic order at the end of the plateau regime, after which the harmonic generation starts to decrease rapidly. We define the cutoff harmonic order $q_{\max }$ as the highest integer for which the corresponding harmonic intensity is, on a logarithmic scale, still at least half as strong as in the plateau regime. This definition is slightly different from that adopted by some authors.

We have found that the cutoff harmonic observed in all harmonic spectra from a two-level model can be determined by an empirical formula expressed in terms of the Rabi frequency $\Omega_{0}=-\mathbf{E}_{0} \cdot \mu \boldsymbol{\epsilon}_{x} / \hbar$ and of the atomic or transition frequency $\omega_{0}$. The maximum order of harmonics before the cutoff is given by

$$
q_{\max } \simeq\left(\omega_{0} / 2+2 \Omega_{0}\right) / \omega_{L}
$$

Figure 2 shows a curve fit between our formula given by Eq. (6) above and data from numerical studies of two-level harmonic spectra. This formula is in good agreement with 


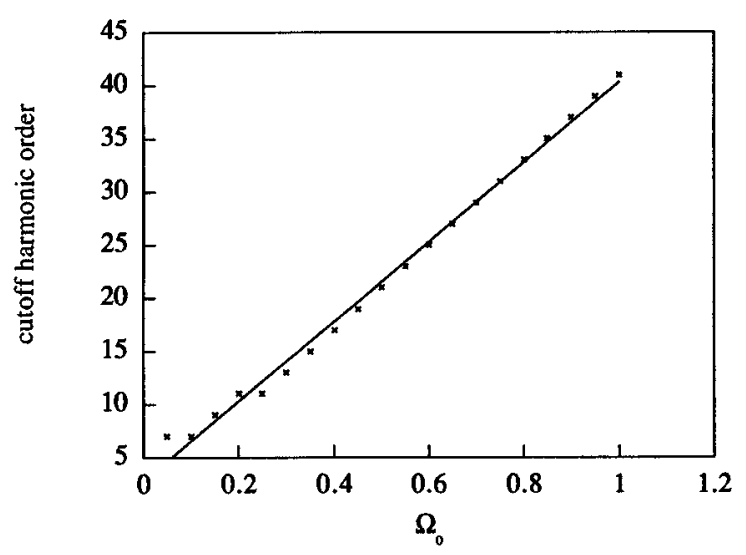

FIG. 2. The cutoff harmonic order as a function of the Rabi frequency $\Omega_{0}$, in atomic units, for a two-level system with atomic transition frequency $\omega_{0}=0.3 \mathrm{a} . \mathrm{u}$. is represented as dots for the laser frequency $\omega_{L}=0.0516$ a.u. The line corresponds to the values obtained by the cutoff law formula defined in Sec. IV. The cutoff is taken to be located at approximately the highest integer for which the corresponding harmonic intensity is, on a logarithmic scale, still at least half as strong as in the plateau regime.

Sundaram and Milonni [7], who predicted a proportionality of $q_{\max }$ to $\Omega_{0}$ for the case when the contribution due to $\omega_{0}$ is negligible. Moreover, it is similar to the one obtained for a two-level system also in the strong field limit but when using a perturbation theory in $\omega_{0} / \omega_{L}$ (see Ivanov and Corkum in [15]). In such a case $\left(\omega_{0}<\omega_{L}\right)$ the maximum frequency in the spectrum is $q_{\max } \simeq 2 \Omega_{0} / \omega_{L}$. We see that in our case $\left(\omega_{0}>\omega_{L}\right)$ the cutoff law is corrected by a term $\omega_{0} / 2 \omega_{L}$, analogous to the ionization potential term in the well-known tunneling case. Our law differs slightly from that obtained by Kaplan and Shkolnikov [12] as a consequence of a minor difference in the definition of the cutoff position.

The linear dependence of the maximum harmonic order on the field amplitude is characteristic of two-level systems and is very different from the dependence $q_{\max } \propto E_{0}^{2}$ found in the case of real atoms in the tunneling regime. We can es- tablish a relation between the cases of the two-level system and of a real atom, and find similarities in the cutoff laws when making the correspondence:

$$
\begin{gathered}
\omega_{0} / 2 \leftrightarrow I_{p}, \\
2 \Omega_{0} \leftrightarrow 3 U_{p} .
\end{gathered}
$$

The ionization energy $I_{P}$ of the ground state for real atoms and the energy difference of the two levels $\hbar \omega_{0}$ represent the maximal potential energies that can be released via transitions from the lowest continuum state (for the real atom) or from the highest bound state (for the two-level atom) to the ground state. This would lead to a correspondence of $I_{P}$ to $\hbar \omega_{0}$. We believe, however, that the factor $1 / 2$ in front of $\hbar \omega_{0}$ is due to the fact that harmonics are generated on a time scale of several Rabi oscillations between the two levels. Then the potential energy is on average between both states and only $\hbar \omega_{0} / 2$ can be released by a transition to the ground state.

The correspondence of the two kinetic energy contributions $2 \hbar \Omega_{0}$ and $3 U_{P}$ is somewhat more subtle. When we apply the laser field $\mathbf{E}=\mathbf{E}_{0} \sin \left(\omega_{L} t\right)$ with Rabi frequency $\Omega_{0}=E_{0} \mu / \hbar$ each level can be envisaged as being split by $\hbar \Omega_{0}$ via the dynamical Stark shift. The maximal energy difference between the two sets of dressed states then increases by just $2 \hbar \Omega_{0}$. A clearer picture of the relationship between the ponderomotive energy and the Rabi interaction energy may be obtained by considering the displacement (and hence dipole moment) of an electron by tunneling from the ionic core. If we imagine a laser field which exceeds the Coulomb field, then the displacement of the electron from the core may be obtained by solving the classical equation of motion of an electron in just the laser field $E_{0} \sin \left(\omega_{L} t\right)$. This results in the usual peak ponderomotive displacement $x=e E_{0} / m \omega_{L}^{2}$, and an effective dipole $\mu_{C}=e^{2} E_{0} / m \omega_{L}^{2}$. If we loosely identify this tunneling dipole with the quantum dipole of the two-level system, $\mu \cong \mu_{C}$, then we find the correspondence

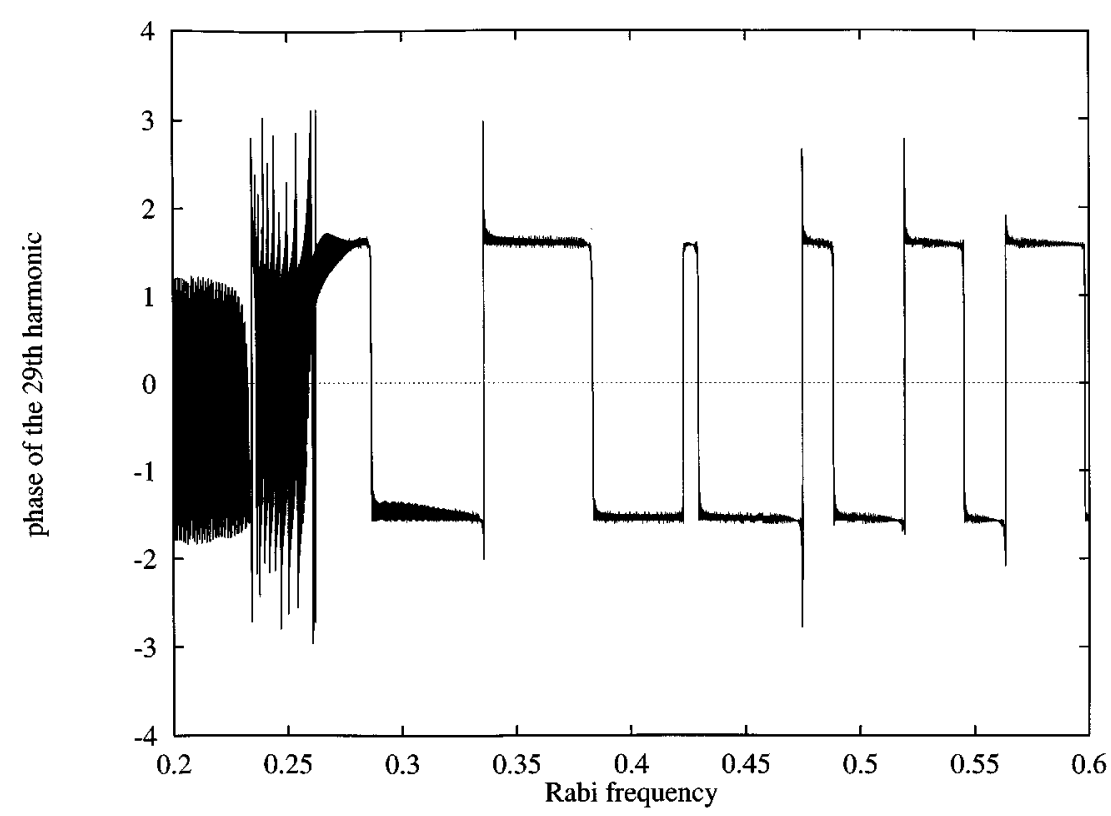

FIG. 3. The phase of the 29th harmonic $\varphi_{q}$, when defined between $[-\pi, \pi]$, as a function of the Rabi frequency $\Omega_{0}$ varying from 0.2 a.u. to 0.6 a.u. The atomic transition frequency is $\omega_{0}=0.3$ a.u. and the laser frequency is $\omega_{L}=0.0258$. The laser was applied in square laser pulses of a duration of 64 cycles and the Rabi frequency corresponding to the cutoff is approximately equal to 0.3 a.u. 


$$
2 \hbar \Omega_{0}=2 \mu E_{0} \cong 2 \frac{e^{2} E_{0}^{2}}{m \omega^{2}}=8 U_{P} .
$$

The energy $8 U_{P}$ is the energy the classical electron can maximally acquire in a laser field. Our simple comparison has not led to the "proper"' prefactor 3.17, which arises from the fact that, in the tunneling recollision model, not the maximal dipole but that at the moment of recollision is relevant. This could be included of course but does not seem relevant for our two-level model. To emphasize our point, we do not claim there is a ponderomotive energy in the two-level model, but merely that the dynamical quantities of both different models $\Omega_{0}$ and $U_{P}$ can be associated once the relevant physical quantities, here the electric dipoles, are compared.

\section{THE PHASE OF THE HARMONICS}

We are interested in the variation of the phase of the dipole moment $\varphi_{q}$ [see Eq. (3)] with respect to the laser field amplitude for a particular harmonic. When the Rabi frequency is increased, a harmonic located initially in the cutoff region joins the plateau once $\Omega_{0}>\Omega_{\text {cutoff }}$ is fulfilled. We consider the dependence of the phase on the Rabi frequency, in both the cutoff and plateau regions.

The results presented here correspond to an atomic frequency $\omega_{0}=0.3$ a.u., a driving frequency $\omega_{L}=0.0258$ a.u., and a Rabi frequency varying from 0.2 to 0.6 a.u. The laser field was applied in square pulses of 64 cycles. The behavior of the phase of the 29th harmonic as a function of $\Omega_{0}$ is presented in Fig. 3 for $\varphi_{q}$ between $[-\pi, \pi]$. The respective Rabi frequency at the cutoff, $\Omega_{\text {cutoff }}$, is approximately equal to 0.3 a.u.

We have performed similar studies for several harmonics of different orders. We clearly note, even within the restriction to the period of $2 \pi$, that the behavior of the phases changes dramatically when we move from the cutoff region to the plateau region. In the cutoff regime the phase appears to be rather scattered, while in the plateau regime we have distinct "jumps" of the phase between essentially $\pi / 2$ and $-\pi / 2$ at various particular Rabi frequencies. These jumps can be associated with avoided crossings of Floquet states which occur at precisely these particular Rabi frequencies. We have checked this quantitatively following the wellknown Floquet approach [21]. However, to enable us to concentrate on explaining the underlying physics, we have decided to display in Fig. 4 a qualitative picture of the main features of the Floquet states as a function of the Rabi frequency. The Floquet states are designated by $|x, n\rangle$, where $x \in\{e, g\}$ represents an atomic state and $n$ is the FloquetFourier index, which can be a positive or negative integer. The energy of these states varies as a function of the Rabi frequency as indicated in a periodical pattern in the vertical direction (with respect to the laser frequency $\omega_{L}$ ). The horizontal direction also shows a certain degree of periodicity as we have alternately crossings and anticrossings (or avoided crossings) of the Floquet states. The latter, where population transfers can take place, are indicated by an arrow, and their width increases slightly when increasing the Rabi frequency. At the anticrossings, we find that the hyper-Raman lines [22] (given by transitions of the type $|e\rangle \rightarrow|g\rangle$ and $|g\rangle \rightarrow|e\rangle$ ) match in frequency the harmonic transitions (given by tran-

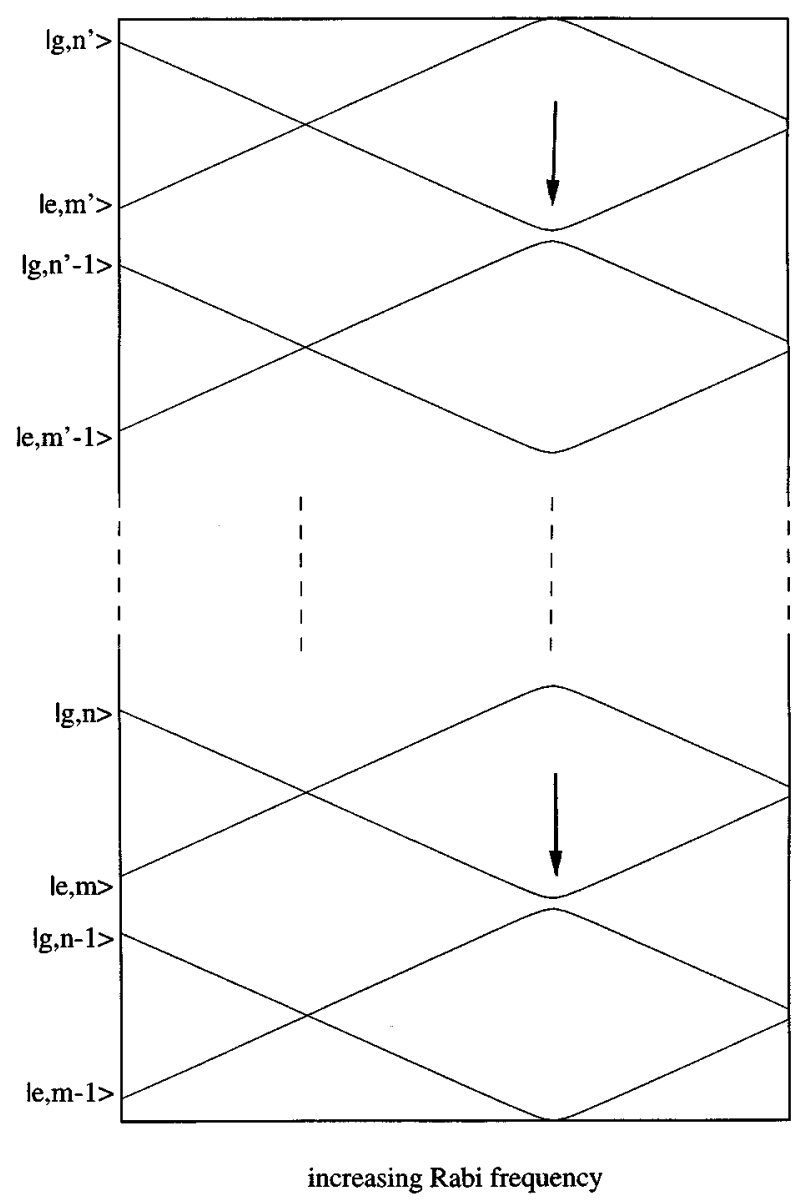

FIG. 4. Schematic diagram of Floquet state energies as a function of an increasing Rabi frequency in the case of the two-level system, for the same parameters as in Fig. 3. The range over which the Rabi frequency is increased is equal to 0.06 a.u. Crossings and anticrossings are visible for particular Rabi frequencies. The arrows indicate the locations of the anticrossings, at which population transfers can take place.

sitions of the type $|g\rangle \rightarrow|g\rangle$ and $|e\rangle \rightarrow|e\rangle)$, so that couplings and interferences occur of the two kinds of transitions leading to the same harmonic frequency. In addition to the involvement of hyper-Raman lines, we also have at these anticrossings a coupling of the $|g\rangle \rightarrow|g\rangle$ and $|e\rangle \rightarrow|e\rangle$ transitions leading to the same harmonic. Furthermore, there may be a transfer of population between these two groups of states giving rise to this harmonic transition at the anticrossings; these couplings and transfers of population can only occur at anticrossings of the Floquet states. We shall address this issue elsewhere.

Let us now consider that the phase varies regularly, i.e., that $d_{q}$ rotates around the trigonometric circle in only one direction, and cease restricting ourselves to a single period of $2 \pi$ of the phase. We then find that the phase decreases, that is, that $d_{q}$ rotates in the negative sense along the trigonometric circle. The corresponding phase behavior for the 29th harmonic is shown in Fig. 5. We find that the phase decreases linearly in the cutoff region as $\sim-1.5 \times 10^{4} \Omega_{0}$ or $\sim-3.87 \times 10^{2} \Omega_{0} / \omega_{L}$, but there is an abrupt change in slope around $\Omega_{\text {cutoff }}$ after which the phase decreases more steeply but still linearly, following $\sim-2.9 \times 10^{4} \Omega_{0}$ or $\sim-7.48 \times 10^{2} \Omega_{0} / \omega_{L}$. We have performed this analysis for 


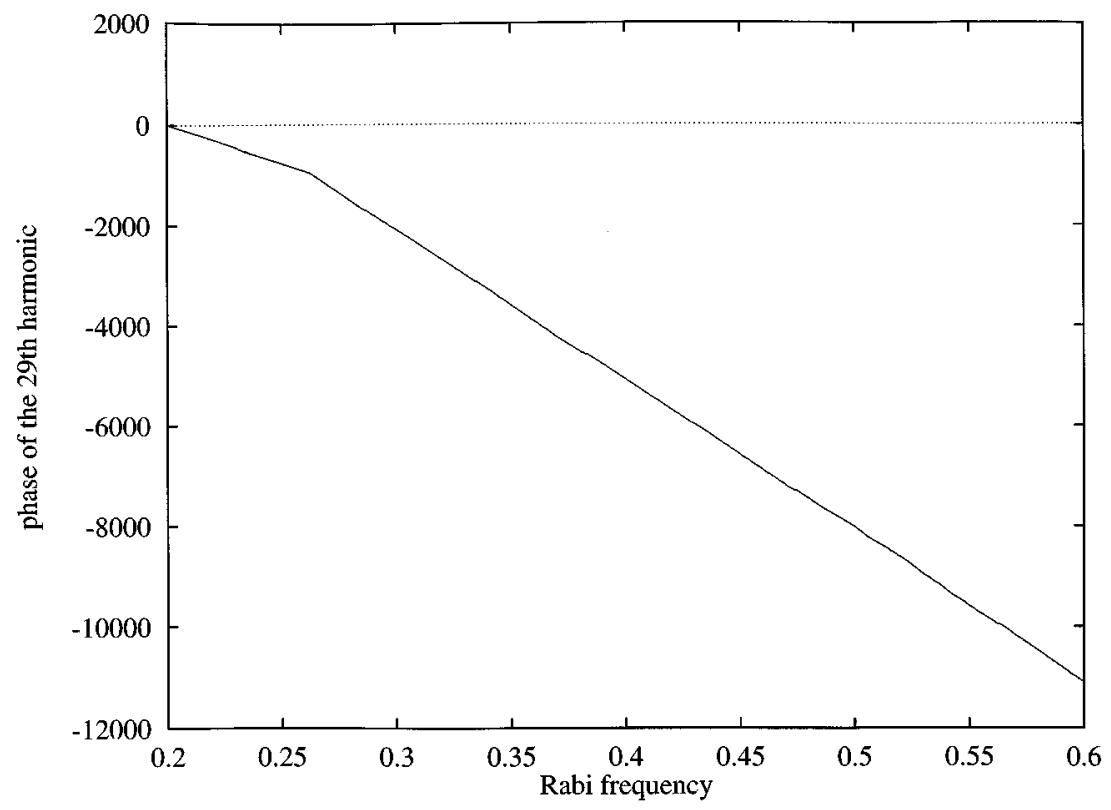

FIG. 5. The phase of the 29th harmonic $\varphi_{q}$, when considered as a monotonous function of the Rabi frequency. The parameters are the same as in Fig. 3.

various harmonic orders. We found that all the respective phases present the same behavior, and moreover have identical slopes, depending upon whether the particular harmonic is in the plateau or in the cutoff region. The ratio of the phase slope in the plateau region to the slope in the cutoff region is given in all cases by $\eta \sim 1.9$. This is in good agreement with the results presented by Salières et al. in [18] for the atom modeled by a Volkov continuum plus a discrete state tunneling model, in that we find a similar linear behavior both in the cutoff and plateau regions, with an abrupt change of slope around the cutoff Rabi frequency. Moreover, the ratio in [18] between the two slopes is given by $\eta \sim 1.81$, and hence has a value which is very close to the one found in the case of the two-level system. Let us note here that the differences between the absolute values of the slopes presented here and those obtained in [18] are due to the fact that the parameters which govern the excitation of the system are quite different. In particular, the number of laser pulse cycles involved in our model is much larger than that considered in [18]; and indeed we found that the magnitude of the phase slope is directly proportional to the number of cycles of the laser pulse excitation: the phase seems to accumulate during the Rabi oscillations, becoming more negative as the laser pulse lasts longer; we are currently investigating this issue further. However, the ratio $\eta$ does not depend on the number of cycles.

The implications of the reduced phase dependence in the cutoff regime are rather significant for phase matching as has been thoroughly investigated by Lewenstein and co-workers $[18,19]$. In a focused laser pulse geometry the atoms are affected by different laser intensities such that the harmonics from the various atoms still contribute with different phases even for forward detection. This undesirable effect is reduced if there is a decreased dependence of the phase of the harmonics on the driving Rabi frequency. Therefore, from the point of view of phase coherence, harmonic generation is more efficient in the cutoff regime. Antoine et al. [23] have discussed how a set of high harmonics can be recombined in an appropriately phased superposition to generate attosecond pulse trains (see also [24,25]). Clearly the relative phases between the harmonics will be crucial for the realization of such a proposal.

\section{CONCLUSIONS}

In this paper we have investigated the dependence of the phase of high harmonics on the Rabi frequency for a strongly driven two-level system. We were able to reproduce results similar to those obtained in the case of models which allow ionization and for which the difference in the phase variation with the laser intensity is believed to be related to the difference in time spent by the electron before recombining. In a two-level model one cannot talk of a free electron and recollisions play no role. Therefore we see that the above explanation, relevant for real atoms, is not unique in explaining the phase variation; ours explains the linear dependence of the harmonic phase with the Rabi frequency as well as the characteristic change of slope around the cutoff. We have shown that this change of slope arises due to phase jumps at avoided crossings of Floquet states in the plateau regime. This issue will be discussed elsewhere in another context. We believe that the sudden change in slope of the phase is actually an intrinsic and generic property of the interaction of the laser field with a nonlinear system, in the same way as the plateau and cutoff are generic. All these common characteristics between the response of a real atom and that of the two-level system, when driven by strong laser fields, should be part of a fundamental behavior of strongly driven nonlinear systems.

After completion of this manuscript, our attention was drawn to the work of Liu and Clark [26], who addressed related issues for the one-dimensional Rochester potential.

\section{ACKNOWLEDGMENTS}

We would like to thank D. G. Lappas, M. Lewenstein, P. Salières, and R. Taieb for valuable discussions. We acknowledge financial support from the European Community, the DRET (French Ministry of Defence), and the UK Engineering and Physical Sciences Research Council. 
[1] J. H. Eberly, Q. Su, and J. Javanainen, Phys. Rev. Lett. 62, 881 (1989); J. Opt. Soc. Am. B 6, 1289 (1989); J. H. Eberly, Q. Su, J. Javanainen, K. C. Kulander, B. W. Shore, and L. RosoFranco, J. Mod. Opt. 36, 829 (1989).

[2] A. L'Huillier, L. A. Lompré, G. Mainfray, and C. Manus, in Atoms in Intense Laser Fields, edited by M. Gavrila (Academic Press, Boston, 1992), p. 139.

[3] The whole field of superintense field-atom interaction is reviewed in Atoms in Intense Laser Fields (Ref. [27]).

[4] K. Burnett, V. C. Reed, and P. L. Knight, J. Phys. B 26, 561 (1993).

[5] M. Lewenstein, P. Balcou, M. Yu. Ivanov, A. L'Huillier, and P. Corkum, Phys. Rev. A 49, 2117 (1994).

[6] W. Becker, S. Long, and J. K. McIver, Phys. Rev. A 50, 1540 (1994); note that their model has also been used to determine the phase by Macklin et al. in Ref. [16].

[7] B. Sundaram and P. W. Milonni, Phys. Rev. A 41, 6571 (1990).

[8] K. Rzążewski and J. H. Eberly, J. Mod. Opt. 39, 795 (1992).

[9] L. Plaja and L. Roso, J. Mod. Opt. 40, 793 (1993).

[10] J. H. Eberly and M. V. Fedorov, Phys. Rev. A 45, 4706 (1992).

[11] D. G. Lappas, M. V. Fedorov, and J. H. Eberly, Phys. Rev. A 47, 1327 (1993).

[12] A. E. Kaplan and P. L. Shkolnikov, Phys. Rev. A 49, 1275 (1994).

[13] F. I. Gauthey, C. H. Keitel, P. L. Knight, and A. Maquet, Phys.
Rev. A 52, 525 (1995), and references therein.

[14] J. L. Krause, K. J. Schafer, and K. C. Kulander, Phys. Rev. Lett. 68, 3535 (1992); Phys. Rev. A 45, 4998 (1992); P. B. Corkum, Phys. Rev. Lett. 71, 1994 (1993).

[15] M. Yu. Ivanov and P. B. Corkum, Phys. Rev. A 48, 580 (1993).

[16] J. J. Macklin, J. D. Kmetec, and C. L. Gordon III, Phys. Rev. Lett. 70, 766 (1993).

[17] A. L'Huillier and Ph. Balcou, Phys. Rev. Lett. 70, 774 (1993).

[18] P. Salières, A. L'Huillier, and M. Lewenstein, Phys. Rev. Lett. 74, 3776 (1995).

[19] M. Lewenstein, P. Salières, and A. L'Huillier, Phys. Rev. A 52, 4747 (1995).

[20] K. Burnett, V. C. Reed, J. Cooper, and P. L. Knight, Phys. Rev. A 45, 3347 (1992).

[21] Jon H. Shirley, Phys. Rev. 138, B979 (1965).

[22] T. Millack and A. Maquet, J. Mod. Opt. 40, 2161 (1993), and references therein.

[23] P. Antoine, A. L'Huillier, and M. Lewenstein, Phys. Rev. Lett. 77, 1234 (1996).

[24] M. Ivanov, P. B. Corkum, T. Zuo, and A. D. Bandrauk, Phys. Rev. Lett. 74, 2933 (1995); S. E. Harris, J. J. Macklin, and T. W. Hänsch, Opt. Commun. 100, 487 (1993).

[25] M. Protopapas, D. G. Lappas, C. H. Keitel, and P. L. Knight, Phys. Rev. A 53, R2933 (1996).

[26] W. -C. Liu and C. W. Clark, Phys. Rev. A 53, 3582 (1996). 